\title{
Erratum: Quantum oscillations in the kinetic energy density: Gradient corrections from the Airy gas [Phys. Rev. B 90, 075139 (2014)]
}

\author{
A. Lindmaa, A. E. Mattsson, and R. Armiento \\ (Received 21 January 2017; published 9 February 2017)
}

DOI: 10.1103/PhysRevB.95.079902

An important result of our paper is expressed in Eq. (23), i.e., the exact positive kinetic energy density for the Airy gas (AG). However, in this equation there is a misprint. The correct equation should read

$$
\tau_{0}^{\mathrm{AG}}(\zeta)=\frac{1}{20 \pi}\left\{2\left(1-\zeta^{3}\right) \mathrm{Ai}^{2}(\zeta)+\zeta \operatorname{Ai}(\zeta) \mathrm{Ai}^{\prime}(\zeta)+2 \zeta^{2}\left[\mathrm{Ai}^{\prime}(\zeta)\right]^{2}\right\}
$$

This misprint does not affect any other result or conclusion of the paper. In the derivation of gradient expansion Eq. (31) and for all subsequent results, the correct equation, Eq. (1) of this Erratum has been used. 